\title{
Search for the Charmonia Dissociation in Lattice QCD
}

\author{
H. Ohno ${ }^{1}$, T. Umeda ${ }^{2}$ and K. Kanaya ${ }^{1}$ \\ (WHOT-QCD Collaboration) \\ ${ }^{1}$ Graduate School of Pure and Applied Sciences, University of Tsukuba, Tsukuba, \\ Ibaraki 305-8571, Japan \\ ${ }^{2}$ Yukawa Institute for Theoretical Physics, Kyoto University, Kyoto 606-8502, Japan \\ E-mail: ohno@het.ph.tsukuba.ac.jp
}

\begin{abstract}
Dissociation of charmonia in hot QCD is studied on a quenched anisotropic lattice using standard plaquette gauge action and $O(a)$ improved Wilson fermion action. Simulations are carried out at temperatures in the range $0.88 T_{C}$ to $2.3 T_{C}$. We calculate effective masses and Bethe-Salpeter wave functions for the ground state $(1 \mathrm{~S}, 1 \mathrm{P})$ and some excited states $(2 \mathrm{~S}, 2 \mathrm{P})$ with variational analysis. To distinguish between bound states and scattering states, we apply two methods: First, we compare effective masses for charmonium correlation functions under different spatial boundary conditions. Because the scattering states are sensitive to the boundary conditions, finite volume effects are expected when a charmonium dissociates. Second, we study if the wave functions show a sign of scattering states which will be characterized by a broad wave function. We find no clear evidences of dissociation for the charmonium states up to $2.3 T_{C}$ so far.
\end{abstract}




\section{Introduction}

To understand properties of quark-gluon-plasma (QGP) formed by a heavy ion collision, it is important to study the behavior of heavy quarkonia above the critical temperature $T_{C}$. According to the $\mathrm{J} / \psi$ suppression scenario [1], $\mathrm{J} / \psi$ may dissolve in the deconfinement phase because of the color screening effect and the suppression of $\mathrm{J} / \psi$ signals can be a evidence of QGP formation in heavy ion collisions at RHIC and LHC. Recently, a sequential $\mathrm{J} / \psi$ suppression scenario [2] in which dissociation of $\chi_{c}(1 \mathrm{P})$ and $\Psi(2 \mathrm{~S})$ indirectly contributes to the suppression of total $\mathrm{J} / \psi$ yield is proposed. Therefore, it is important to study dissociation of chamonia above the deconfinement temperature $T_{C}$.

In lattice QCD, to investigate the dissociation temperature of chamonia, spectral functions of chamonia were studied with the Maximum Entropy Method (MEM). These studies indicate that $\mathrm{S}$ wave ground states survive up to about $1.5 T_{C}[3,4,5,6,7]$, on the other hand, $\mathrm{P}$ wave ground states dissolve just above $T_{C}[5,6,7]$. There is no detailed study about the dissociation of excited charmonia. These results look consistent with the sequential $\mathrm{J} / \psi$ suppression scenario.

However, there are following two problems. First, the dissociation signal of $\mathrm{P}$ wave charmonia in previous studies may be misleading because of the constant mode contributions in meson correlation functions at finite temperature. A study of the constant mode in quenched QCD [8] suggested that especially $\mathrm{P}$ wave correlation functions receive sizable contributions of constant modes above $T_{C}$, which may disturb extracting signals we are really interested in. Actually, when such contributions are subtracted out from the meson correlation functions, $\mathrm{P}$ wave charmonia showed to survive up to $1.4 T_{C}$, in contrast to the conclusion of previous studies [8]. Second, MEM results are known to depend on the choice of the default model. It is not clear what kind of default model is proper for meson spectral functions above $T_{C}$. Therefore, a crosscheck without Bayesian-type analyses is needed.

What can we expect on the lattice when a charmonium dissolve? First of all, it should be kept in mind that the spectral functions on a lattice with finite extent consist of discrete spectra only, both below and above $T_{C}$. Below $T_{C}$, we will have discrete peaks corresponding to the ground state and some excited states in the charmonium spectral function. When all charmonium bound states fully dissolve above $T_{C}$, the peaks corresponding to these bound states should vanish and the discrete peaks corresponding to the $c-\bar{c}$ scattering states will appear. Furthermore, we expect that bound state wave functions have spatially localized shapes, while wave functions of $c-\bar{c}$ scattering states extend spatially to large distances.

In this paper, we report on our study of charmonia dissociation. Our approach consists of a combination of robust methods without resorting to Bayesian-type analyses. First, we apply the midpoint subtraction method developed in [8] to separate out the constant mode contributions from meson correlation functions. In principle, when a high precision data of the correlation function is available up to large distances, the constant 
Table 1. The parameter $A_{i}$ of the smearing functions.

\begin{tabular}{|c||c|c|c|c|c|c|}
\hline$i$ & 1 & 2 & 3 & 4 & 5 & 6 \\
\hline$A_{i}$ & 0.02 & 0.05 & 0.10 & 0.15 & 0.20 & 0.25 \\
\hline
\end{tabular}

mode can be identified by a MEM analysis too. In practice, however, with current accuracy and the range of sensible data, we think that it is safer to explicitly subtract out the contribution of the constant mode from the correlation functions. Second, in order to extract both ground state and excited state masses, we adopt multi-state variational analysis [9]. Furthermore, we calculate the Bethe-Salpeter wave functions of these states from the spatial correlation function between $c-\bar{c}$ quarks to distinguish the $c-\bar{c}$ bound states from scattering states: We investigate the spatial boundary condition dependence of energy spectra at finite volume [10] and the spatial distribution of wave functions [11]. The energy of scattering states should depend on its relative momentum which is quantized according to the spatial size and boundary conditions. On the other hand, the spectrum of the bound states will not change against such exchange of boundary conditions. Moreover, the wave functions for a bound state should be compact, while those for a scattering state should extend to large distances and change its shape depending on the spatial lattice size. Combining these tests, we may examine if a charmonium dissociates.

\section{Multi-state variational analysis}

The meson correlation matrix in Euclidian space-time is defined by

$$
C_{i j}(t) \equiv \sum_{\vec{x}}\left\langle O_{i}^{\Gamma}(\vec{x}, t) O_{j}^{\Gamma}(\overrightarrow{0}, 0)^{\dagger}\right\rangle
$$

with $\Gamma=\gamma_{5}, \gamma_{i}, \mathbf{1}(i=1,2,3)$ for pseudo-scalar (Ps), vector (Ve) and scalar (Sc) channels, respectively. Here $\left\{O_{i}^{\Gamma} \mid i=1,2, \cdots N_{\text {state }}\right\}$ are smeared meson operators defined by

$$
O_{i}^{\Gamma}(\vec{x}, t) \equiv \sum_{\vec{y}, \vec{z}} \omega_{i}(\vec{y}) \omega_{i}(\vec{z}) \bar{q}(\vec{x}+\vec{y}, t) \Gamma q(\vec{x}+\vec{z}, t),
$$

with Gaussian smearing functions $\omega_{i}(\vec{x}) \equiv \exp \left(-A_{i}|\vec{x}|^{2}\right)$. Table 1 shows our choice of the smearing parameters $A_{i}$.

According to [8], the constant mode contribution becomes large in the deconfined phase because the constant mode is due to wraparound contributions of single quark propagators which are suppressed in the confined phase. To separate out the constant mode contribution from meson correlation functions, we study the midpoint subtracted correlation functions $\bar{C}(t)$ defined by

$$
\begin{aligned}
\bar{C}(t) & \equiv C(t)-C\left(N_{t} / 2\right) \\
& =\left(c_{0}+\sum_{k=1}^{\infty} c_{k} \cosh \left[m_{k}\left(t-\frac{N_{t}}{2}\right)\right]\right)-\sum_{k=0}^{\infty} c_{k}
\end{aligned}
$$




$$
=2 \sum_{k=1}^{\infty} c_{k} \sinh ^{2}\left[\frac{m_{k}}{2}\left(t-\frac{N_{t}}{2}\right)\right] .
$$

Here $C(t)$ is the matrix of $C_{i j}(t)$ defined in (1), $c_{0}$ is a contribution of the constant mode and $N_{t}$ is the temporal lattice size.

We can extract ground and excited states in $\bar{C}$ by solving the generalized eigenvalue problem,

$$
\bar{C}(t) \vec{v}_{k}=\lambda_{k}\left(t ; t_{0}\right) \bar{C}\left(t_{0}\right) \vec{v}_{k} \quad\left(k=1,2, \cdots, N_{\text {state }}\right) .
$$

Then effective masses of the diagonalized states $M_{k}^{\text {eff }}$ are given by

$$
\lambda_{k}\left(t ; t_{0}\right)=\frac{\sinh ^{2}\left[\frac{M_{k}^{\text {eff }}}{2}\left(t-\frac{N_{t}}{2}\right)\right]}{\sinh ^{2}\left[\frac{M_{k}^{\text {eff }}}{2}\left(t_{0}-\frac{N_{t}}{2}\right)\right]} .
$$

Next, the BS wave function of the $k$-th meson state $M_{k}$ is defined by,

$$
\psi_{k}(\vec{r}) \equiv \sum_{\vec{x}}\left\langle\bar{q}(\vec{x}) \Gamma q(\vec{x}+\vec{r}) \mid M_{k}\right\rangle
$$

In order to calculate the wave function, we define the spatial correlation function between $c-\bar{c}$ quarks as,

$$
\begin{aligned}
C_{i}(\vec{r}, t) & \equiv \sum_{\vec{x}}\left\langle\bar{q}(\vec{x}, t) \Gamma_{\mathrm{snk}} q(\vec{x}+\vec{r}, t) O_{i}^{\Gamma_{\mathrm{src}}}(\overrightarrow{0}, 0)^{\dagger}\right\rangle \\
& =\sum_{k=0}^{\infty} c_{k}^{(i)} \psi_{k}(\vec{r}) \cosh \left[m_{k}\left(t-\frac{N_{t}}{2}\right)\right]
\end{aligned}
$$

Here $c_{k}^{(i)} \equiv\left\langle M_{k} \mid O_{i}^{\Gamma_{\operatorname{src}}}(\overrightarrow{0}, 0)^{\dagger}\right\rangle$ is the overlap between the $k$-th meson state and the smeared source operator $O_{i}^{\Gamma_{\mathrm{src}}}$. To study $\mathrm{S}$ waves, we adopt $\Gamma_{\mathrm{src}}=\Gamma_{\mathrm{snk}}=\gamma_{5}$ and $\gamma_{i}$ for Ps and Ve charmonia, respectively. For Sc charmonium in $\mathrm{P}$ wave, we use a derivative source $\Gamma_{\mathrm{src}}=\gamma_{i}\left(\overleftarrow{\partial_{i}}-\overrightarrow{\partial_{i}}\right)$ to enhance the signal, with $\Gamma_{\mathrm{snk}}=\gamma_{i}$

To separate out the constant mode, we define the midpoint subtracted spatial correlation function $\overrightarrow{\vec{C}}(\vec{r}, t)$ by

$$
\overrightarrow{\vec{C}}(\vec{r}, t) \equiv \vec{C}(\vec{r}, t)-\vec{C}\left(\vec{r}, N_{t} / 2\right) .
$$

Here $\vec{C}(\vec{r}, t)$ is a vector whose $i$-th component is defined in (7). Finally, the diagonalized wave function which is normalized at $\overrightarrow{r_{0}}$ is given by

$$
\Psi_{k}\left(\vec{r} ; \overrightarrow{r_{0}}\right) \equiv \frac{\overrightarrow{\vec{C}}(\vec{r}, t) \cdot \vec{v}_{k}}{\overline{\vec{C}}\left(\overrightarrow{r_{0}}, t\right) \cdot \vec{v}_{k}} .
$$

\section{Numerical results}

\subsection{Lattice setup}

We study quenched QCD with the anisotropy $\xi \equiv a_{s} / a_{t}=4$. The gauge action is the standard plaquette action with $\beta=6.10$ and a bare anisotropy parameter $\gamma_{G}=3.2108$. 
Table 2. The number of gauge configurations.

\begin{tabular}{|c||c|c|c|}
\hline & $N_{s}=16$ & $N_{s}=20$ & $N_{s}=32$ \\
\hline local operator & 800 & 800 & 200 \\
\hline derivative operator & 300 & 300 & 200 \\
\hline
\end{tabular}
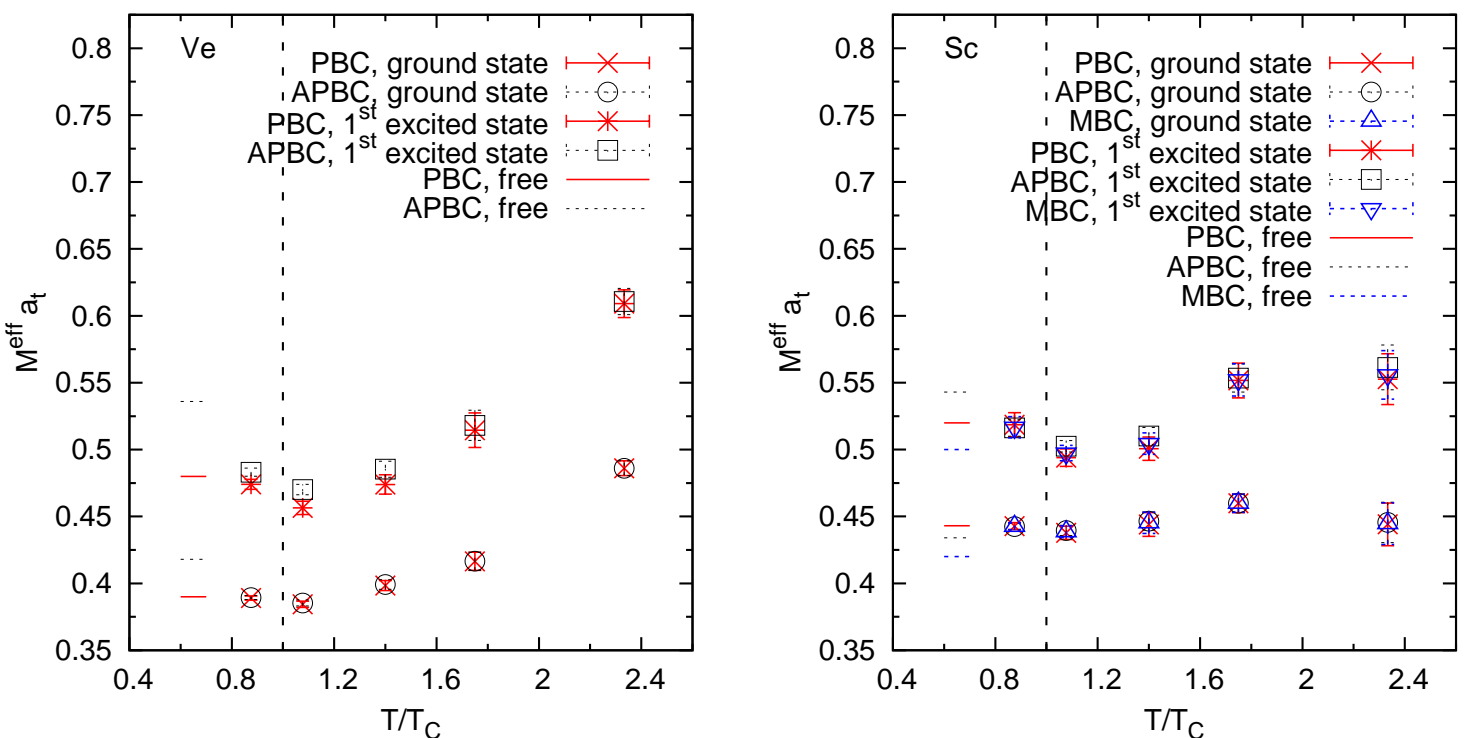

Figure 1. The effective masses for Ve (left) and Sc (right) charmonia. Cross, circle and triangle symbols indicate the effective masses of the ground state with $\mathrm{PBC}$, $\mathrm{APBC}$ and MBC, respectively (see text for the definition of MBC). Asterisk, square and inverted triangle symbols indicate the effective masses of the first excited state with PBC, APBC and MBC, respectively. Short bars shown near the left end of each figure indicate the boundary condition dependence of energy spectra for the case of free quarks.

For quarks, we adopt $O(a)$ improved Wilson fermion action with tadpole-improved treelevel clover coefficients. Our actions and their parameters are the same as those in [12] except for the choice of the Wilson parameter, $r=1$. The spatial lattice spacing is $a_{s}^{-1}=2.030(13) \mathrm{GeV}$. Simulations are performed on $N_{s}^{3} * N_{t}$ lattice, with $N_{s}=16,20$ and 32 and $N_{t}=32,26,20,16$ and 12, which correspond to $T=\left(N_{t} a_{t}\right)^{-1}=0.88 T_{c}$, $1.1 T_{c}, 1.4 T_{c}, 1.8 T_{c}$ and $2.3 T_{c}$ respectively. (Critical temperature $T_{c}$ corresponds to $N_{t} \simeq 28$.) The number of gauge configurations is shown in Table 2. We adopt the Coulomb gauge fixing.

\subsection{Effective masses}

In Fig. 1, we compare the effective masses under different boundary conditions for the ground state and the first excited state of Ve and Sc channel. We choose three boundary 
conditions, i.e. periodic boundary condition (PBC), anti-periodic boundary condition (APBC) and mixed boundary condition (MBC). Here MBC is defined by APBC in the $x$-direction combined with PBC in $y$ and $z$-directions. At zero temperature, the ground state and the first excited state of Ve channel correspond to $J / \psi(1 \mathrm{~S})$ and $\psi(2 \mathrm{~S})$ respectively, and the ground state of $\mathrm{Sc}$ channel corresponds to $\chi_{c 0}(1 \mathrm{P})$. The effective masses are calculated by the multi-state variational analysis with $4 \times 4$ correlation matrix on the $20^{3} \times N_{t}$ lattice. Here we choose smeared operators with the parameter $A_{3}, A_{4}, A_{5}$ and $A_{6}$ of Table 1 to obtain the largest overlap with the ground state and the first excited state.

To see a typical magnitude of the boundary-condition dependence in the effective masses, we study the energy spectra for the case of free quark in a box of $(2 \mathrm{fm})^{3}$, assuming that the charm quark mass is $1.3 \mathrm{GeV}$. Results are shown by short bars around the left end of each plot in Fig.1. (Only relative differences in the vertical coordinates are relevant for the bars.) For ground state $\mathrm{P}$ waves, we obtain the largest gap between $\mathrm{PBC}$ and MBC. Therefore, we may naively expect a mass shift of about $200 \mathrm{MeV}$ (500 $\mathrm{MeV}$ ) for a ground (first exited) state if the charmonium fully dissolve.

We find no evidence of mass shift with such magnitudes, for neither the ground state nor the first excited state, up to a quite high temperature of about $2.3 T_{c}$. We note that the effective masses increase with temperature. This may be caused by the small temporal extent at high temperatures, which makes it is more difficult to extract asymptotic signals reliably. At least, however, the lack of a mass shift under the change of the boundary condition suggests that the contribution of scattering states, if it exists, is negligibly-small up to $2.3 T_{C}$. Results for Ps charmonia are similar. This suggests that these charmonia bound state persist up to $2.3 T_{c}$.

\subsection{Wave functions}

We calculate the wave functions corresponding to the mass eigenstates diagonalized by the multi-state variational analysis discussed in the previous subsection. When a charmonium dissociates, corresponding wave function should extend to large distances and show a sensitive dependence on the spatial size of the box. On the other hand, when the charmonium persist as a bound state, the wave function will be compact and not so sensitive to the spatial lattice size. Figure 2 shows the wave functions defined by (9) for the ground state and the first excited state of Ve and Sc channel at $2.3 T_{c}$, obtained on three different spatial extent lattices of $32^{3}, 20^{3}$ and $16^{3}$. We find that, for all charmonia we study, the spatial size dependence is small, and the wave functions are compact. It suggests that the bound states are still dominant and we thus find no sign of $c-\bar{c}$ scattering states up to $2.3 T_{c}$, in accordance with the observation discussed in the previous subsection. 

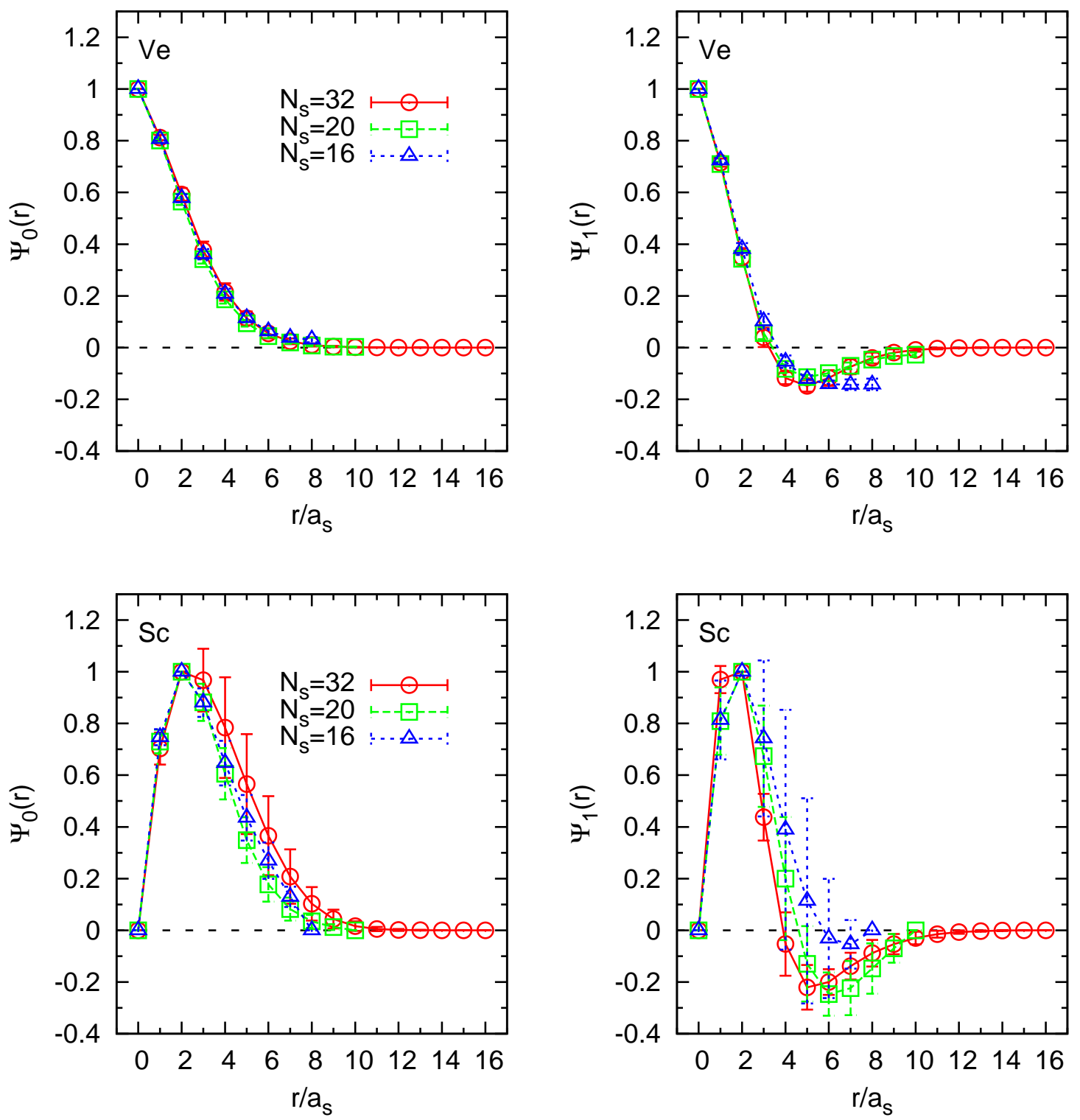

Figure 2. The wave functions for Ve (top) and Sc (bottom) charmonia at $2.3 T_{C}$. Horizontal axis is the spatial distance in the $x$-direction. Left panels show the results for ground states and right panels show those of the first excited states. Circle, square and triangle symbols indicate data obtained on $N_{s}=32,20$ and 16 lattices.

\section{Conclusions}

We investigated if charmonia dissociates in high temperature QCD on quenched anisotropic lattices. Adopting the multi-state variational analysis to extract ground and first excited states, we examined spatial boundary condition dependence of effective masses and investigated the shape and spatial size dependence of wave functions for these states. From these studies, we found no sign of scattering states nor clear evidence of dissociation, both for the ground and first exited states of charmonia in Ps, Ve and Sc 
channels up to the temperature of about $2.3 T_{c}$, so far.

\section{Acknowledgments}

We thank the members of the WHOT-QCD Collaboration for innumerable discussions and suggestions. This work is in part supported by Grants-in-Aid of the Japanese Ministry of Education, Culture, Sports, Science and Technology (Nos. 17340066 and 19549001). Numerical calculations were performed on supercomputers at RCNP, Osaka University.

\section{References}

[1] T. Matsui and H. Satz, Phys. Lett. B 178, 416 (1986)

[2] S. Digal, P. Petreczky and H. Satz, (2001) [hep-ph/0110406]; F. Karsch, D. Kharzeev and H. Satz, Phys. Lett. B 63775 (2006) [hep-ph/0512239]

[3] T. Umeda, K. Nomura and H. Matsufuru, Eur. Phys. J. C 39S1 9 (2005) [hep-lat/0211003]

[4] M. Asakawa and T. Hatsuda, Phys. Rev. lett. 92, 012001 (2004) [hep-lat/030803]

[5] S. Datta, F. Karsch, P. Petreczky and I. Wetzorke, Phys. Rev. D 69, 094507 (2004) [hep-lat/031203]

[6] A. Jackovac, P. Petreczky, K. Petrov and A. Velytsky, Phys. Rev. D 75, 014506 (2007) [heplat/0611017]

[7] G. Aarts, C. Allton, M. B. Oktay, M. Peardon and J. I. Skullerud, Phys. Rev. D 76, 094513 (2007) [hep-lat/0705.2198]

[8] T. Umeda, Phys. Rev. D 75, 094502 (2007) [hep-lat/0701005]

[9] M. Lüscher and U. Wolff, Nucl. Phys. B 339, 222 (1990)

[10] H. Iida, T. Doi, N. Ishii, H. Suganuma and K. Tsumura, Phys. Rev. D 74, 074502 (2006) [heplat/0602008]

[11] T. Umeda, R. Katayama, O. Miyamura and H. Matsufuru, Int. J. Mod. Phys. A 162215 (2001) [hep-lat/0011085]

[12] H. Matsufuru, T. Onogi and T. Umeda, Phys. Rev. D 64, 114503 (2001) [hep-lat/0107001] 